\title{
Asymptotic Analysis of the parton branching equation at LHC Energies
}

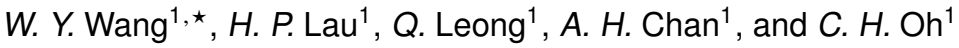 \\ ${ }^{1}$ Physics Department, National University of Singapore, Singapore
}

\begin{abstract}
An asymptotic solution to the QCD parton branching equation is derived using the method of Laplace transformation and saddle point approximation. The distribution is applied to charged particle multiplicity distributions in proton-proton collisions at $\sqrt{s}=$ $0.9,2.36$, and $7 \mathrm{TeV}$ for $|\eta|<0.5,1.0,1.5,2.0,2.4$, and $8 \mathrm{TeV}$ for $|\eta|<0.5,1.0,1.5$, as well as $13 \mathrm{TeV}$ data for $|\eta|<0.8$ and 2.5 .
\end{abstract}

\section{Introduction}

The charged particle multiplicity distribution is a global observable of the final state of high energy collisions. The shape of the distribution offers information on the dynamics of particle production processes as well as any correlation in particle production. Photon counting distributions in optical experiment and theory has shown that the independent production of photons lead to a Poisson distributed counting distribution. In the context of high energy proton-proton $(p p)$ and electron-positron $\left(e^{+} e^{-}\right)$collisions, the charged-particle multiplicity distributions are most often described using the Negative Binomial Distribution (NBD) [1] and even the double NBD [2]. The NBD describes cascades (or showers) of particles originating from an initial number of ancestor particles produced after the collision [3].

The total multiplicity distribution of partons within a jet [4] can be expressed as Markov branching processes of quarks $(q)$ and gluons $(g)$ [5]. Considering the processes $g \rightarrow g+g, q \rightarrow q+g, g \rightarrow q+q$, and $g \rightarrow g+g+g$ with average branching probabilities of $A, \tilde{A}, B$, and $C$ respectively, the QCD parton branching differential equation for producing $m$ quarks and $n$ gluons after branching [6-8] is given as:

$$
\begin{aligned}
\frac{\partial P_{m, n}(t)}{\partial t}= & -\tilde{A} m P_{m, n}(t)+\tilde{A} m P_{m, n-1}(t) \\
& -A n P_{m, n}(t)+A(n-1) P_{m, n-1}(t) \\
& -B n P_{m, n}(t)+B(n+1) P_{m, n+1}(t) \\
& -C n P_{m, n}(t)+C(n-2) P_{m, n-2}(t),
\end{aligned}
$$

where

$$
t=\frac{6}{11 N_{c}-2 N_{f}} \ln \left[\frac{\ln \left(Q^{2} / \mu^{2}\right)}{\ln \left(Q_{0}^{2} / \mu^{2}\right)}\right]
$$

^e-mail: w.y.wang@nus.edu.sg 
and $Q$ is the initial parton invariant mass, $Q_{0}$ is the hadronisation mass, $\mu$ is a QCD mass scale of a few $\mathrm{GeV}, N_{c}$ is the number of colours, and $N_{f}$ is the number of flavours.

The above equation yields the Generalised Multiplicity Distribution (GMD) [6-8] as a possible exact solution in the case where $B=C=0$. Given an initial $m$ and $k^{\prime}$ number of quarks $(q)$ and gluons $(g)$, the probability of obtaining $n$ final state particles is given by the GMD:

$$
P_{G M D}\left(n ; p, k, k^{\prime}\right)=\frac{\Gamma(n+k)}{\Gamma\left(n-k^{\prime}+1\right) \Gamma\left(k^{\prime}+k\right)}(1-p)^{n-k^{\prime}}(p)^{k^{\prime}+k},
$$

where $k=m \tilde{A} / A, p=\exp (-A t)=\left(k^{\prime}+k\right) /(\bar{n}+k)$, and $\bar{n}$ is the average number of observed final state particles. Special cases of the GMD are the NBD when $k^{\prime}=0$, and Fury-Yule Distribution [9] when $k=0$. Furthermore, the NBD converges to the PD for $k \rightarrow \infty$.

However, an exact solution including all the processes in equation 1 has never been derived. In this paper, an asymptotic solution is obtained using the method of Laplace transformation and the saddle point approximation. The result corrects an error by A. H. Chan and C. K. Chew [10] in the use of the Stirling's formula. The resulting asymptotic multiplicity distribution is then applied to measurements of charged particle multiplicity distributions in $p p$ collisions by the ALICE [11], ATLAS [12], and CMS [13] collaborations at the LHC.

\section{Asymptotic solution of the QCD parton branching equation}

Using a continuous-variable approximation, where $n$ is replaced by $x \in \mathbb{R}$ and performing a Laplace transform with initial condition, equation 1 becomes

$$
\begin{aligned}
-P(x, t=0)+s P^{*}(x, s)= & -A^{\dagger} P^{*}(x, s)+A^{\dagger} P^{*}(x-1, s) \\
& -A x P^{*}(x, s)+A(x-1) P^{*}(x-1, s) \\
& -B x P^{*}(x, s)+B(x+1) P^{*}(x+1, s) \\
& -C x P^{*}(x, s)+C(x-2) P^{*}(x-2, s),
\end{aligned}
$$

with $A^{\dagger}=\tilde{A} m$.

When $x>k^{\prime}$, the initial condition ensures that $P\left(x>k^{\prime}, t=0\right)=0$. In the case where $x=k^{\prime}$,

$$
\begin{gathered}
-P\left(x=k^{\prime}, t=0\right)+s P^{*}\left(k^{\prime}, s\right)=-\left(A k^{\prime}+A^{\dagger}+B k^{\prime}+C k^{\prime}\right) P^{*}\left(k^{\prime}, s\right), \\
P^{*}\left(k^{\prime}, s\right)=\frac{1}{s+A^{\dagger}+(A+B+C) k^{\prime}} .
\end{gathered}
$$

To further simplify equation 4 when $x>k^{\prime}$, let $\ln \left(P^{*}(x, s)\right)=L(x)$ and approximate $L(x+1) \sim L(x)$ and $L(x-2) \sim L(x-1)$ as we expect large number of gluons to be created at high energies. Equation 4 then becomes

$$
\begin{aligned}
s e^{L(x)}= & -\left(A x+A^{\dagger}+B x+C x\right) e^{L(x)} \\
& +A(x-1) e^{L(x)-L^{\prime}(x)}+A^{\dagger} e^{L(x)-L^{\prime}(x)} \\
& +B(x+1) e^{L(x)}+C(x-2) e^{L(x)-L^{\prime}(x)}
\end{aligned}
$$

where a Taylor expansion has been made for all the $L(x-1)$ terms about $L(x)$ up to the first differential coefficient. 
Rearranging, the equation

$$
-\frac{\partial L(x)}{\partial x}=\ln \left[\frac{s+A^{\dagger}-B+(A+C) x}{(A+C) x-A+A^{\dagger}-2 C}\right]
$$

can be obtained.

To simplify the analysis, let

$$
\begin{array}{r}
\alpha=A^{\dagger}-B, \\
\beta=A+C, \\
\gamma=A^{\dagger}-A-2 C, \\
D=A+B+C .
\end{array}
$$

Integrating equation 8, and letting $L_{1}\left(k^{\prime}, s\right)=\ln P^{*}\left(x=k^{\prime}, s\right)$ (Eq. 6),

$$
L_{1}(x, s)=-\int_{k^{\prime}}^{x} \ln \left(\frac{s+\alpha+\beta \omega}{\gamma+\beta \omega}\right) d \omega-\ln \left(s+A^{\dagger}+D k^{\prime}\right),
$$

A better approximation can then be obtained by expanding the Taylor series up to the second term,

$$
-\frac{\partial L(x)}{\partial x}+\frac{1}{2} \frac{\partial^{2} L(x)}{\partial x^{2}}=\ln \left(\frac{s+\alpha+\beta x}{\gamma+\beta x}\right) .
$$

Differentiating equation 8 , and substituting into equation 11 ,

$$
-\frac{\partial L(x)}{\partial x}=\ln \left(\frac{s+\alpha+\beta x}{\gamma+\beta x}\right)-\frac{\beta}{2}\left(\frac{1}{\gamma+\beta x}-\frac{1}{s+\alpha+\beta x}\right)
$$

which on integrating gives

$$
L_{2}(x, s)=-\int_{k^{\prime}}^{x} \ln \left(\frac{s+\alpha+\beta \omega}{\gamma+\beta \omega}\right) d \omega+\ln \left[\frac{(\gamma+\beta x)^{1 / 2}\left(s+\alpha+\beta k^{\prime}\right)^{1 / 2}}{\left(\gamma+\beta k^{\prime}\right)^{1 / 2}(s+\alpha+\beta x)^{1 / 2}\left(s+A^{\dagger}+D k^{\prime}\right)}\right] .
$$

The required asymptotic solution is obtained by the inverse Laplace transform

$$
P_{2}(x, t)=\frac{1}{2 \pi i} \int_{C-i \infty}^{C+i \infty} g(s) e^{s t-f(s)} d s
$$

where

$$
g(s)=\frac{(\gamma+\beta x)^{1 / 2}\left(s+\alpha+\beta k^{\prime}\right)^{1 / 2}}{\left(\gamma+\beta k^{\prime}\right)^{1 / 2}(s+\alpha+\beta x)^{1 / 2}\left(s+A^{\dagger}+D k^{\prime}\right)}
$$

and

$$
f(s)=\int_{k^{\prime}}^{x} \ln \left(\frac{s+\alpha+\beta \omega}{\gamma+\beta \omega}\right) d \omega .
$$

The integral of equation 14 is expected to be dominated by the highest saddle point at $s=s_{0}$ for large $t$. Using the saddle point approximation, the asymptotic solution is given by

$$
P_{2}(x, t)=\frac{g\left(s_{0}\right) e^{s_{0} t-f\left(s_{0}\right)}}{\sqrt{-2 \pi f^{\prime \prime}\left(s_{0}\right)}} .
$$


Substituting the corresponding expressions for $g\left(s_{0}\right), f\left(s_{0}\right)$, and $f^{\prime \prime}\left(s_{0}\right)$, equation 17 becomes

$$
P_{2}(x, t)=\frac{\left[\frac{(\gamma+\beta x)\left(s_{0}+\alpha+\beta k^{\prime}\right)}{\left(\gamma+\beta k^{\prime}\right)\left(s_{0}+\alpha+\beta x\right)}\right]^{1 / 2} \frac{1}{s_{0}+A^{\dagger}+D k^{\prime}} e^{s_{0} t-\int_{k^{\prime}}^{x} \ln \left(\frac{s_{0}+\alpha+\beta \omega}{\gamma+\beta k^{\prime}}\right) d \omega}}{\sqrt{2 \pi \int_{k^{\prime}}^{x} \frac{1}{\left(s_{0}+\alpha+\beta \omega\right)^{2}} d \omega}},
$$

which can be further evaluated by looking for the stationary function of the exponential term in the above equation

$$
\frac{\partial}{\partial s_{0}}\left[e^{s_{0} t-\int_{k^{\prime}}^{x} \ln \left(\frac{s_{0}+\alpha+\beta \omega}{\gamma+\beta k^{\prime}}\right) d \omega}\right]=0
$$

This gives the expression

$$
s_{0}=\frac{\left(x-k^{\prime}\right) \beta}{1-e^{-\beta t}}-\alpha-\beta x
$$

which by letting $k=\frac{\gamma}{\beta+1}$, we obtain

$$
\begin{aligned}
P_{2}(x, t) & =\frac{e^{-t\left(\alpha+\beta k^{\prime}+\beta\right)}\left(1-e^{-\beta t}\right)^{x-k^{\prime}}}{\frac{B}{\beta}\left(1+k^{\prime}\right)\left(1-e^{-\beta t}\right)+\left(x-k^{\prime}\right) e^{-\beta t}} \times\left(x-k^{\prime}\right) \\
& \times \frac{(k+x-1)^{k+x-1 / 2}}{(2 \pi)^{1 / 2}\left(x-k^{\prime}\right)^{x-k^{\prime}+1 / 2}\left(k+k^{\prime}-1\right)^{k+k^{\prime}-1 / 2}} .
\end{aligned}
$$

For large values of $x$, the Stirling's approximation is given by $x !=\frac{x^{x+1 / 2}}{e^{x}}(2 \pi)^{1 / 2}$. Taking note that $x !=\Gamma(x+1)$ and replacing $x$ by $n$, the final form of the asymptotic solution to the QCD parton branching equation is obtained as

$$
P(n, t)=\frac{e^{-t\left(\alpha+\beta k^{\prime}+\beta\right)}\left(1-e^{-\beta t}\right)^{n-k^{\prime}}}{\frac{B}{\beta}\left(1+k^{\prime}\right)\left(1-e^{-\beta t}\right)+\left(n-k^{\prime}\right) e^{-\beta t}} \times\left(n-k^{\prime}\right) \times \frac{\Gamma(n+k)}{\Gamma\left(n-k^{\prime}+1\right) \Gamma\left(k^{\prime}+k\right)} .
$$

Replacing $\alpha, \beta, \gamma$, and $D$ with their corresponding expressions in equation 9 and letting $A=A t$, $A^{\dagger}=A^{\dagger} t, B=B t$, and $C=C t$,

$$
\begin{array}{r}
P(n)=\frac{e^{-\left(A^{\dagger}+A-B+C\right)} e^{-(A+C) k^{\prime}}\left(1-e^{-(A+C)}\right)^{n-k^{\prime}}}{\frac{B}{A+C}\left(1+k^{\prime}\right)\left(1-e^{-(A+C)}\right)+\left(n-k^{\prime}\right) e^{-(A+C)}} \\
\quad \times\left(n-k^{\prime}\right) \times \frac{\Gamma\left(n+\frac{A^{\dagger}-C}{A+C}\right)}{\Gamma\left(n-k^{\prime}+1\right) \Gamma\left(k^{\prime}+\frac{A^{\dagger}-C}{A+C}\right)} .
\end{array}
$$

With the last substitution, the branching probabilities $A, A^{\dagger}, B, C$ are now reinterpreted as the branching probabilities scaled by the QCD evolution parameter. The ratios between the branching probabilities (e.g. $A^{\dagger} / A$ ) remain fixed regardless of the scale.

Applied to charged particle multiplicity data, equation 23 represents the probability of obtaining $n$ charged particles from the hadronisation of gluons in QCD jets, via the branching processes associated with the parameters $A, A^{\dagger}, B$, and $C$. 

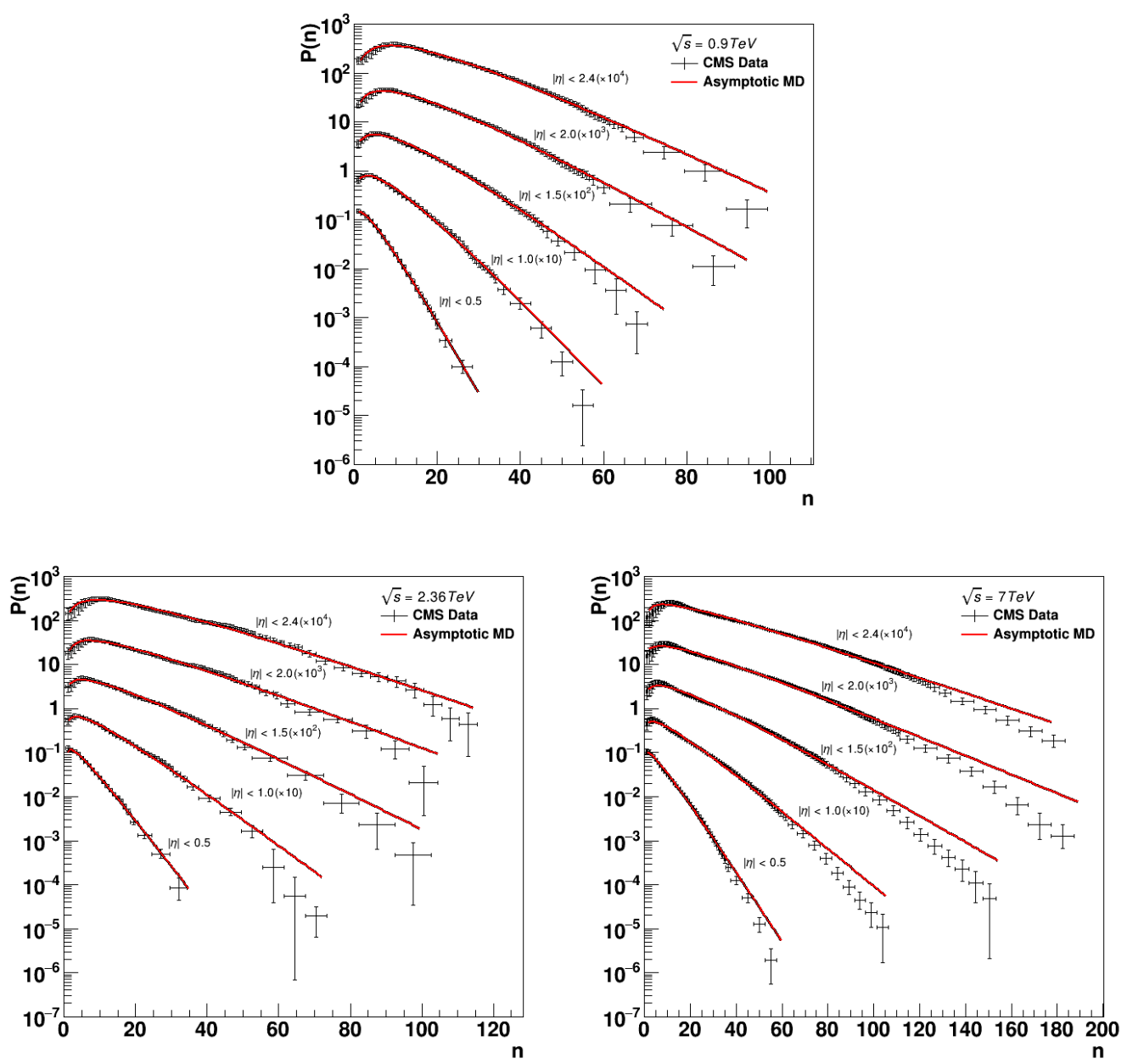

Figure 1. The asymptotic multiplicity distribution compared to CMS charged particle multiplicity data at (top) $\sqrt{s}=0.9$, (bottom left) 2.36 , and (bottom right) $7 \mathrm{TeV}$ for all pseudorapidity windows $|\eta|<0.5,1.0,1.5,2.0,2.4$. The values for successively larger pseudorapidities are scaled by factors of 10 for clarity in presentation.

\section{Results}

Figure 1 shows charged particle multiplicity distributions in $p p$ collisions for five pseudorapidity windows $|\eta|<0.5,1.0,1.5,2.0$, and 2.4 as measured by the CMS collboration at $\sqrt{s}=0.9,2.36$, and $7 \mathrm{TeV}$. Comparison is made with the best fit asymptotic multiplicity distribution. The corresponding parameter values are given in table 1.

Similarly, figure 2 (top) shows charged particle multiplicity distributions for $|\eta|<0.5,1.0$, and 1.5 at $\sqrt{s}=8 \mathrm{TeV}$ measured by the ALICE collaboration. Figure 2 (bottom) shows the same distributions for $|\eta|<0.8$ and 2.5 at $\sqrt{s}=13 \mathrm{TeV}$ measured by the ATLAS collaboration.

The asymptotic multiplicity distribution is found to describe data from CMS collaboration well. However it is noted that there is increasing difficulty in describing the tail of the distribution as the 

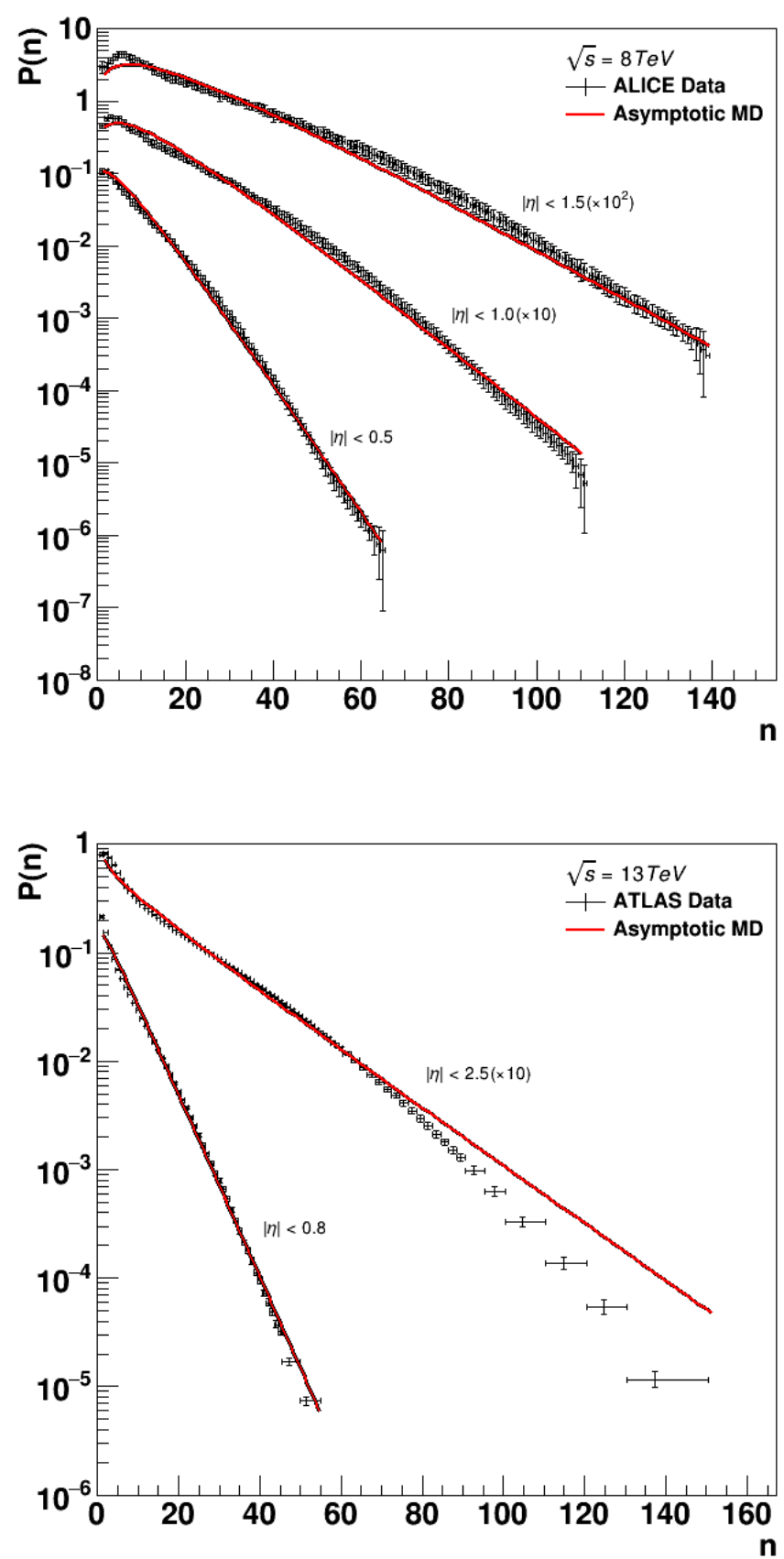

Figure 2. The asymptotic multiplicity distribution compared to charged particle multiplicity distributions measured by (top) ALICE at $\sqrt{s}=8 \mathrm{TeV}$ for $|\eta|<0.5,1.0,1.5$ and by (bottom) ATLAS at $\sqrt{s}=13 \mathrm{TeV}$ for $|\eta|<0.8,2.5$. The values for successively larger pseudorapidities are scaled by factors of 10 for clarity in presentation. 
pseudorapidity range increases. This suggests that the asymptotic multiplicity distribution is insufficient to describe all the processes involved in $p p$ collisions and the subsequent fragmentation. At $\sqrt{s}=8 \mathrm{TeV}$ the asymptotic multiplicity distribution appears to describe the tail of the distribution well. However, it poorly models the data at mid-multiplicity $(n \sim 70)$ and low-multiplicity $(n \sim 10)$ regimes. At $\sqrt{s}=13 \mathrm{TeV}$, the data is well modelled for $|\eta|<0.8$ but described poorly for $|\eta|<2.5$, where the tail of the distribution is overestimated from $n \sim 70$.

Referring to table 1 , the values of $k^{\prime}, B$ and $C$ generally tend to 0 or some small value for centreof-mass energies of up to $8 \mathrm{TeV}$. This implies that quark pair creation and the four-gluon vertex occur at a relatively insignificant rate during the branching process. Quark bremsstrahlung and gluon fission dominate the branching process, and the entire process occurs with no initial gluons. Hence at the above centre-of-mass energies, charged particle multiplicities are still better described by the NBD $\left(k^{\prime}=0\right)$. However the non-zero $k^{\prime}$ values at $\sqrt{s}=13 \mathrm{TeV}$ seem to suggest that more initial gluons are produced at higher centre-of-mass energies.

\section{Conclusion}

From the QCD parton branching equation, the asymptotic multiplicity distribution is derived using the method of Laplace transform and saddle point approximation. Revision of the derivation from previous work is done with the correct Stirling's formula. Starting with an initial number of gluons $k^{\prime}$, the distribution describes the probability of producing $n$ gluons via four processes that contribute to the overall quark and gluon distribution inside QCD jets, with each process occuring with a different probability.

Table 1. Parameter values for the asymptotic multiplicity distribution.

\begin{tabular}{|c|c|c|c|c|c|c|c|}
\hline$\sqrt{s}(\mathrm{TeV})$ & $|\eta|$ & $k^{\prime}$ & $A^{\dagger}$ & $A$ & $B$ & $C$ & $\chi^{2 / d o f}$ \\
\hline \hline \multirow{5}{*}{$0.9(\mathrm{CMS})$} & 0.5 & 0 & 2.1 & 1.13 & 0.0 & 0.06 & $0.42 / 17$ \\
\cline { 2 - 8 } & 1.0 & 0 & 3.1 & 1.59 & 0.0 & 0.06 & $10.43 / 34$ \\
\cline { 2 - 8 } & 1.5 & 0 & 3.8 & 1.92 & 0.0 & 0.05 & $16.40 / 46$ \\
\cline { 2 - 8 } & 2.0 & 0 & 4.3 & 2.15 & 0.1 & 0.06 & $17.21 / 56$ \\
\cline { 2 - 8 } & 2.4 & 0 & 4.6 & 2.29 & 0.1 & 0.06 & $24.91 / 62$ \\
\hline \multirow{5}{*}{$2.36(\mathrm{CMS})$} & 0.5 & 0 & 2.2 & 1.44 & 0.0 & 0.04 & $1.92 / 17$ \\
\cline { 2 - 8 } & 1.0 & 0 & 3.2 & 1.95 & 0.0 & 0.04 & $15.32 / 34$ \\
\cline { 2 - 8 } & 1.5 & 0 & 3.6 & 2.32 & 0.1 & 0.05 & $14.20 / 44$ \\
\cline { 2 - 8 } & 2.0 & 0 & 3.9 & 2.54 & 0.2 & 0.1 & $23.68 / 54$ \\
\cline { 2 - 8 } & 2.4 & 0 & 4.6 & 2.65 & 0.1 & 0.05 & $35.94 / 62$ \\
\hline & 0.5 & 0 & 2.4 & 1.73 & 0.00 & 0.04 & $20.60 / 35$ \\
\cline { 2 - 8 } & 1.0 & 0 & 3.4 & 2.27 & 0.00 & 0.05 & $77.55 / 64$ \\
\cline { 2 - 8 } & 1.5 & 0 & 4.0 & 2.61 & 0.00 & 0.06 & $140.55 / 89$ \\
\cline { 2 - 8 } & 2.0 & 0 & 4.2 & 2.97 & 0.00 & 0.02 & $112.71 / 109$ \\
\cline { 2 - 7 } & 2.4 & 0 & 4.3 & 3.16 & 0.04 & 0.02 & $97.66 / 121$ \\
\hline \multirow{3}{*}{8 (ALICE) } & 0.5 & 0 & 2.47 & 1.65 & 0.00 & 0.00 & $55.79 / 60$ \\
\cline { 2 - 8 } & 1.0 & 0 & 3.60 & 2.15 & 0.00 & 0.05 & $206.26 / 106$ \\
\cline { 2 - 7 } & 1.5 & 0 & 4.3 & 2.44 & 0.00 & 0.10 & $286.34 / 134$ \\
\hline \multirow{3}{*}{13 (ATLAS) } & 0.8 & 0.997 & 0.17 & 1.70 & 0.00 & 0.02 & $45.63 / 42$ \\
\cline { 2 - 7 } & 2.5 & 0.859 & 0.00 & 2.85 & 0.00 & 0.00 & $336.50 / 76$ \\
\hline
\end{tabular}


The asymptotic multiplicity distribution is applied to charged particle multiplicity distribution data at $\sqrt{s}=0.9,2.36,7,8$, and $13 \mathrm{TeV}$ from the ALICE, ATLAS, and CMS collaborations. The parameters $k^{\prime}, A^{\dagger}, A, B$, and $C$ are estimated and the result is found to describe the observed multiplicity well at $\sqrt{s}=0.9,2.36$, and $7 \mathrm{TeV}$ well except for the tail of the distribution. The parameter $k^{\prime}$ reduces to 0 (yielding NBD), suggesting that branching processes up to $7 \mathrm{TeV}$ are dominated by gluon fission and quark bremsstrahlung with negligible initial gluon densities.

The asymptotic multiplicity distribution is found to be inadequate in modelling observed multiplicities at $\sqrt{s}=8 \mathrm{TeV}$ and more so at $13 \mathrm{TeV}$, where it fails to describe both the mid- and highmultiplicity regions at larger pseudorapidity intervals. This hints at the presence of additional processes which should be accounted for.

\section{Acknowledgements}

The authors would like to thank the National University of Singapore, and the support and helpful discussions with colleagues. We would also like to congratulate the ALICE, ATLAS, and CMS collaboration for their analysis on the multiplicity distributions and thank them for the well organised release of data points to public.

\section{References}

[1] P. Ghosh, Phys. Rev. D 85, 054017 (2012)

[2] ALICE Collaboration (2017), arXiv: 1708.01435v1

[3] A. Giovannini, L. Van Hove, A. Phys. Pol. B 19, 495 (1988)

[4] K. Konishi, A. Ukawa, G. Veneziano, Nucl. Phys. B 157, 45 (1979)

[5] A. Giovannini, Nucl. Phys. B 161, 429 (1979)

[6] C.K. Chew, D. Kiang, H. Zhou, Phys. Lett. B 186, 411 (1987)

[7] A.H. Chan, C.K. Chew, Phys. Rev. D 41, 851 (1990)

[8] A.H. Chan, C.K. Chew, Z. Phys. C 55, 503 (1992)

[9] R.C. Hwa, C.S. Lam, Phys. Lett. B 173, 346 (1986)

[10] A.H. Chan, C.K. Chew, J. Math. Phys. 33, 9 (1992)

[11] ALICE Collaboration, Eur. Phys. J. C 77, 33 (2017)

[12] ATLAS Collaboration, Phys. Lett. B 758, 67 (2016)

[13] CMS Collaboration, JHEP 01, 79 (2011) 